\title{
Maximal cluster sets of $L$-analytic functions along arbitrary curves
}

\author{
L. Bernal-González, A. Bonilla, M.C. Calderón-Moreno \\ and J. A. Prado-Bassas*
}

\begin{abstract}
Let $\Omega$ be a domain in the $N$-dimensional real space, $L$ be an elliptic differential operator, and $\left(T_{n}\right)$ be a sequence whose members belong to a certain class of operators defined on the space of $L$-analytic functions on $\Omega$. It is proved in this paper the existence of a dense linear manifold of $L$-analytic functions all of whose nonzero members have maximal cluster sets under the action of every $T_{n}$ along any curve ending at the boundary of $\Omega$ such that its $\omega$-limit does not contain any component of the boundary. The above class contains all partial differentiation operators $\partial^{\alpha}$, hence the statement extends earlier results due to Boivin, Gauthier and Paramonov, and to the first, third and fourth authors.
\end{abstract}

2000 Mathematics Subject Classification: Primary 30D40. Secondary 30E10, 31B35, 41A30, 47F05.

Key words and phrases: maximal cluster set, $L$-analytic function, dense linear manifold, admissible path, elliptic operator, internally controlled operator.

*The first, third and fourth authors have been partially supported by the Plan Andaluz de Investigación de la Junta de Andalucía FQM-127 and by MCyT Grants BFM200303893-C02-01 and MTM2004-21420-E. The second author has been partially supported by MCYT-FEDER Project no. BFM2002-02098. 


\section{Introduction}

The behavior of the complex-valued functions near the boundary of the domain where they are defined has attracted the attention of many mathematicians. Such a behavior can be considered either globally or restricted to certain subsets (mainly, adequate curves) near the boundary, and is defined by the so-called cluster sets, see below. For background about classical results on cluster sets, we refer to [5] and [9]. Next, we fix some standard notation that will be used throughout this paper.

The symbols $\mathbb{R}, \mathbb{C}, \mathbb{N}, \mathbb{N}_{0}$ will stand for the real line, the complex plane, the set of positive integers, and the set $\mathbb{N} \cup\{0\}$, respectively. If $N \in \mathbb{N}$ then $\mathbb{R}^{N}$ is the $N$-dimensional real space; specially, $\mathbb{C}=\mathbb{R}^{2}$. If $A \subset \mathbb{R}^{N}$ then $A^{0}$ ( $\partial A$, resp.) represents its interior (its boundary, resp.) in $\mathbb{R}^{N}$. In addition, we set $A^{c}:=\mathbb{R}^{N} \backslash A$, as usual. The open ball with center $a \in \mathbb{R}^{N}$ and radius $r>0$-with respect to the euclidean distance $d$ on $\mathbb{R}^{N}$ is $B(a, r)$. By $\Omega$ we denote a domain in $\mathbb{R}^{N}$, that is, a nonempty connected open subset of $\mathbb{R}^{N}$. Moreover, $\Omega^{*}$ will denote the one-point compactification of $\Omega$. A Jordan domain is a domain $\Omega \subset \mathbb{C}$ whose boundary in $\mathbb{C}^{*}$ is a topological image of the unit circle $\{z \in \mathbb{C}:|z|=1\}$. If $A \subset \mathbb{R}^{N}$ and $f$ is a complex-valued function defined on $A$ then $\|f\|_{A}:=\sup _{A}|f|$. For a multiindex $\alpha=\left(\alpha_{1}, \ldots, \alpha_{N}\right) \in \mathbb{N}_{0}^{N}$, we let $|\alpha|=\alpha_{1}+\cdots+\alpha_{N}, \alpha !=\alpha_{1} ! \cdots \alpha_{N}$ !, $x^{\alpha}=x_{1}^{\alpha_{1}} \cdots x_{N}^{\alpha_{N}}$ for $x=\left(x_{1}, \ldots, x_{N}\right) \in \mathbb{R}^{N}$ and $\partial^{\alpha}=\left(\frac{\partial}{\partial x_{1}}\right)^{\alpha_{1}} \cdots\left(\frac{\partial}{\partial x_{N}}\right)^{\alpha_{N}}$.

Let $r, N \in \mathbb{N}$ with $N \geq 2$ and let $L(\xi)=\sum_{|\alpha|=r} a_{\alpha} \xi^{\alpha}\left(\xi \in \mathbb{R}^{N}\right)$ be a fixed homogeneous polynomial of degree $r$ with complex constant coefficients and which satisfies the ellipticity condition $L(\xi) \neq 0\left(\xi \in \mathbb{R}^{N} \backslash\{0\}\right)$. We associate to $L$ the homogeneous elliptic operator of order $r$ given by

$$
L=L(\partial)=\sum_{|\alpha|=r} a_{\alpha} \partial^{\alpha} .
$$

The symbol $\mathcal{L}_{r}^{N}$ stands for the class of all homogeneous elliptic operators of order $r$ in $\mathbb{R}^{N}$ with constant complex coefficients.

If $\Omega \subset \mathbb{R}^{N}$ is a domain and $f: \Omega \rightarrow \mathbb{C}$ is a $C^{\infty}$-function, then $f$ is called $L$-analytic (or L-holomorphic) on $\Omega$ if it satisfies the equation

$$
L(\partial) f=0 \text { on } \Omega .
$$

We denote by $L(\Omega)$ the linear space of $L$-holomorphic functions on $\Omega$ endowed with the compact-open topology. For instance, if $N=2$ then the space of 
$\bar{\partial}$-holomorphic functions is the space $H(\Omega)$ of holomorphic functions in the usual sense, and for arbitrary $N, \Delta$-holomorphic is the same as harmonic in $\mathbb{R}^{N}$ if $\Delta$ denotes the Laplace operator. In general, $L(\Omega)$ turns out to be a Fréchet space (see [12]), which is separable because it is a subspace of $C(\Omega)$, the space of complex continuous functions on $\Omega$, also endowed with the compact-open topology. By an operator on $L(\Omega)$ we mean a continuous linear selfmapping $T: L(\Omega) \rightarrow L(\Omega)$.

Let $\Omega$ be a domain in $\mathbb{R}^{N}, N \geq 2, \Omega \neq \mathbb{R}^{N}$ and $\gamma:[0,1) \rightarrow \Omega$ be a curve in $\Omega$ tending to $\partial \Omega$, that is, $\gamma$ is continuous and, given a compact set $K \subset \Omega$, there is $t_{0}=t_{0}(K) \in(0,1)$ for which $K \cap \gamma\left(\left[t_{0}, 1\right)\right)=\emptyset$. Then the $\omega$-limit (or oscillation set) of $\gamma$ is the set $O s c(\gamma)$ of points in $\partial \Omega$ which are in the closure of $\gamma([0,1))$. Let $b \in \partial \Omega$. Following [3, Section 6$]$, we shall say that a continuous path $\gamma:[0,1] \rightarrow \mathbb{R}^{N}$ is admissible for $\Omega$ with end point $b$ if $\gamma([0,1)) \subset \Omega$ and $\gamma(1)=b$. Note that, in particular, if $\partial \Omega$ has no connected component consisting of a single point then for any admissible path $\gamma$ we have that $O s c(\gamma)$ contains no component of $\partial \Omega$. By abuse of language, we sometimes identify $\gamma=\gamma([0,1))$.

Given a continuous function $f: \Omega \rightarrow \mathbb{C}$ and a curve $\gamma$ in $\Omega$ tending to its boundary, we denote by $\mathcal{C}_{\gamma}(f)$ the cluster set of $f$ along $\gamma$, that is, $\mathcal{C}_{\gamma}(f)=\left\{w \in \mathbb{C}^{*}:\right.$ there exists a sequence $\left\{t_{n}\right\} \subset[0,1)$ such that $t_{n} \rightarrow$ 1 and $f\left(\gamma\left(t_{n}\right)\right) \rightarrow w$ as $\left.n \rightarrow \infty\right\}$.

In [3, Theorem 5], Boivin, Gauthier and Paramonov proved that if $L \in \mathcal{L}_{r}^{N}$ and $\Omega \subset \mathbb{R}^{N}$ is a domain with $\Omega \neq \mathbb{R}^{N}$ such that $\partial \Omega$ has no component consisting of a single point, then there is at least one function $g \in L(\Omega)$ with the property that for each $b \in \partial \Omega$, each admissible path $\gamma$ ending at $b$ and each $\alpha \in \mathbb{N}_{0}^{N}$, one has $C_{\gamma}\left(\partial^{\alpha} g\right)=\mathbb{C}^{*}$, that is, each such cluster set is maximal. On the other hand, in [1, Theorem 2.1] it is shown that if $\Omega$ is a Jordan domain in $\mathbb{C}$ then there is a dense linear manifold $\mathcal{D} \subset H(\Omega)$ all of whose nonzero members $f$ satisfy that the cluster set of $f$ along $\gamma$ is maximal for every curve $\gamma$ in $\Omega$ tending to the boundary with $O s c(\gamma) \neq \partial \Omega$. In this paper, we state a theorem (see Section 4) that unifies and extends largely both results above; our theorem will be valid for a large class of operators on $L(\Omega)$ which will be introduced in Section 2. Section 3 is devoted to present some additional terminology together with a preparatory result about approximation. 


\section{A new class of operators}

We collect in the following definition the adequate class of operators to be used later.

Definition 2.1. Let $r, N \in \mathbb{N}$ with $N \geq 2$ and $\Omega \subset \mathbb{R}^{N}$ be a domain. Assume that $L \in \mathcal{L}_{r}^{N}$ and that $T$ is an operator on $L(\Omega)$. We say that $T$ is internally controlled if given $\varepsilon>0$ and a pair of compact sets $F, G \subset \Omega$ with $F \subset G^{0}$, there exists $\delta=\delta(\varepsilon, F, G)>0$ such that

$$
\left[f \in L(\Omega) \text { and }\|f\|_{G}<\delta\right] \text { implies }\|T f\|_{F}<\varepsilon .
$$

Note that the notion of "internal control" is stronger than the mere continuity. In fact, the former requires in particular the continuity of every mapping $T_{1,2}: X_{1} \rightarrow X_{2}$, where $X_{i}(i=1,2)$ is the normed space consisting of all functions of $L(\Omega)$ under the respective norms $\|\cdot\|_{F},\|\cdot\|_{G}$ and $(F, G)$ is any pair of compact subsets of $\Omega$ with $F \subset G^{0}$, while the continuity of $T$ only requires to fix a compact subset $F$ and find some compact set $G$ in $\Omega$ such that the corresponding mapping $T_{1,2}$ is continuous.

Another property to be imposed in Section 3 on an operator $T$ on $L(\Omega)$ will be that $\operatorname{ran} T(:=T(L(\Omega)))$ contains the constant functions. Of course, this is equivalent to $1 \in \operatorname{ran} T$.

Let us provide some examples of "classical" operators that satisfy some of the above conditions.

Examples 2.2. 1. If $L \in \mathcal{L}_{r}^{N}$ and $\Omega$ is a domain in $\mathbb{R}^{N}$ then from known estimations about the norms of the solutions of a homogeneous elliptic partial differential equation (see for instance [10, pages 188-189, Lemma 1]) it is deduced that each operator $\partial^{\alpha}$ is internally controlled on $L(\Omega)$. On the other hand, in [3, Lemma 3] it is proved that $1 \in \operatorname{ran} L$.

2. In particular, by Example 1 -or by using Cauchy's integral formula for derivatives- we obtain for $N=2$ and $L=\bar{\partial}$ that each derivative operator $D^{n}\left(n \in \mathbb{N}_{0}\right)$ is internally controlled on $H(\Omega)$. Here $D^{0} f=f$ and $D^{n+1} f=$ $\left(D^{n} f\right)^{\prime}$ for every $n \in \mathbb{N}_{0}$ and every $f \in H(\Omega)$. More, if $\Phi(z)=\sum_{n=0}^{\infty} a_{n} z^{n}$ is an entire function of subexponential type (that is, given $\varepsilon>0$ there is a constant $A \in(0,+\infty)$ such that $|\Phi(z)| \leq A \exp (\varepsilon|z|)$ for all $z \in \mathbb{C})$, then its associated (in general, infinite order) differential operator $\Phi(D)=$ $\sum_{n=0}^{\infty} a_{n} D^{n}$ is well defined and internally controlled on $H(\Omega)$. This is easy to see just by taking into account that $\Phi$ is of subexponential type if and only if 
$\lim _{n \rightarrow \infty}\left(n\left|a_{n}\right|^{1 / n}\right)=0$ (see for instance [2]). Moreover, $1 \in \operatorname{ran} \Phi(D)$ as soon as $\Phi \not \equiv 0$. Indeed, let $m=\min \left\{n \in \mathbb{N}_{0}: \Phi^{(n)}(0) \neq 0\right\}$; then $\Phi(D)(h)=1$ if $h(z):=z^{m} / \Phi^{(m)}(0)$.

3. Again in the classical holomorphic case, if $\alpha \in H(\Omega)$ then its associated multiplication operator $M_{\alpha}: f \mapsto \alpha f$ is always internally controlled on $H(\Omega)$. And $1 \in \operatorname{ran} M_{\alpha}$ if and only if $\alpha$ has no zeros in $\Omega$. On the contrary, if $\varphi: \Omega \rightarrow \Omega$ is a holomorphic selfmapping, then the composition operator $C_{\varphi}: f \in H(\Omega) \mapsto f \circ \varphi \in H(\Omega)$ is internally controlled only if $\varphi$ is the identity. We have that $1 \in \operatorname{ran} C_{\varphi}$ for any holomorphic selfmapping $\varphi$.

4. We know that internal control implies continuity. In fact, the former property is strictly stronger. For instance, the Volterra operator $V$ on $H(\mathbb{C})$ given as $(V f)(z)=\int_{0}^{z} f(t) d t$ is easily seen to be not internally controlled. Observe that, in addition, $1 \notin \operatorname{ran} V$ because $(V f)(0)=0$ for every $f \in$ $H(\mathbb{C})$.

5. The family of internally controlled operators is a vector algebra in the space of all operators on $L(\Omega)$, that is, if $\alpha, \beta$ are scalars and $T, S$ are internally controlled operators on $L(\Omega)$, then the operators $\alpha T+\beta S$ and $T \circ S$ are internally controlled too. Indeed, this is evident for $\alpha T+\beta S$. As for the composition $T \circ S$, fix a pair $(F, G)=\left(F_{1}, F_{2}\right)$ of compact sets as well as a number $\varepsilon>0$ as in Definition 2.1. Then choose a compact set $F_{3}$ with $F_{1} \subset F_{3}^{0} \subset F_{3} \subset F_{2}^{0}$, for instance, $F_{3}:=\overline{\bigcup_{x \in F_{1}} B(x, \alpha / 2)}$, where $\alpha=d\left(F_{1}, F_{2}^{c}\right)$. Now apply that $T$ is internally controlled to the pair $\left(F_{3}, F_{2}\right)$ and, finally, apply that $S$ is internally controlled to $\left(F_{1}, F_{3}\right)$.

\section{$3 \quad$ Some auxiliary statements}

Following [4], a relatively closed subset $F$ in $\Omega$ will be called a RothKeldysh-Lavrentiev set, or more simply an $\Omega$-RKL set, if $\Omega^{*} \backslash F$ is connected and locally connected. The following lemma (see [3] and [6]) will reveal useful in the proof of our main result. Note that Arakelian's theorem (see [7]) covers the assertion for $N=2$ in the holomorphic case.

Lemma 3.1. Let $\Omega$ be a domain in $\mathbb{R}^{N}, N \geq 2$ and $F$ be a $\Omega$-RKL set. Assume that $f$ be L-analytic in some neighbourhood of $F$ in $\Omega$. Then, for each $\varepsilon>0$, there exists $g \in L(\Omega)$ such that

$$
\|f-g\|_{F}<\varepsilon .
$$


In order to simplify the notation, we denote by $\mathcal{O}$ the family of domains $\Omega \subset \mathbb{R}^{N}$ such that $\Omega \neq \mathbb{R}^{N}$ and the boundary of $\Omega$ in $\left(\mathbb{R}^{N}\right)^{*}$ has no connected components that consist of a single point. Moreover, for each domain $\Omega$ as before, we denote by $\Gamma(\Omega)$ the family of curves $\gamma \subset \Omega$ tending to the boundary whose $\omega$-limit does not contain any component of the boundary. Then, we establish the following technical lemma.

Lemma 3.2. Assume that $\Omega \in \mathcal{O}$. Then there are three sequences $\left(K_{j}\right)$, $\left(G_{j}\right),\left(F_{j}\right)$ of compact subsets of $\Omega$ satisfying the following properties:

(i) $\Omega=\bigcup_{j=1}^{\infty} K_{j}$ and $K_{j} \subset K_{j+1}^{0}(j \in \mathbb{N})$.

(ii) The $G_{j}$ 's are pairwise disjoint and $G_{j} \subset K_{j}$ for all $j \in \mathbb{N}$.

(iii) For every $j \in \mathbb{N}$ one has that $K_{j} \cap G_{j+1}=\emptyset$ and $K_{j} \cup G_{j+1}$ is an RKL-set.

(iv) $F_{j} \subset G_{j}^{0}(j \in \mathbb{N})$ and every $\gamma \in \Gamma(\Omega)$ intersects all sets $F_{j}$ 's except possibly finitely many of them.

The content of the last lemma is essentially proved in [3, Proof of Theorem 5], so we omit a detailed proof. Suffice it to say that (i), (iii) and the first part of (ii) and of (iv) are obtained in the cited proof, in which each $G_{j}$ is constructed as a thin (closed) neighborhood of $F_{j}$, which in turn is a subset of $K_{j}$. In order to obtain the contention $G_{j} \subset K_{j}$ of (ii), it suffices to replace each $K_{j}$ by a set $\widetilde{K}_{j}$ slightly larger. The sets $K_{j}, G_{j}, F_{j}$ in [3] are closed, but in addition they are constructed by using the level sets of an adequate real analytic function $\Psi$ that satisfies an inequality [3, inequality (6) on page 960], which produces that the above closed sets are bounded; hence they are compact. Finally, the last part of (iv) is shown in [3] for admissible curves, but the same holds for every $\gamma \in \Gamma(\Omega)$ just by using the same argument of [3, page 962].

\section{The main result}

Once the basic terminology and background have been established, we are ready to present our main assertion.

Theorem 4.1. Let $\Omega \in \mathcal{O}$. Assume that $T_{n}: L(\Omega) \rightarrow L(\Omega)(n \in \mathbb{N})$ is a sequence of operators satisfying the following conditions: 
(a) Each $T_{n}$ is internally controlled.

(b) For each $n \in \mathbb{N}$, ran $T_{n}$ contains the constants.

Then there exists a dense linear manifold $\mathcal{D}$ in $L(\Omega)$ such that for every $g \in \mathcal{D} \backslash\{0\}$, every $n \in \mathbb{N}$, and every curve $\gamma \in \Gamma(\Omega)$, we have

$$
\mathcal{C}_{\gamma}\left(T_{n} g\right)=\mathbb{C}^{*}
$$

Proof. From Lemma 3.2, we can choose sequences $\left(K_{j}\right),\left(F_{j}\right),\left(G_{j}\right)$ satisfying properties (i) to (iv) in such lemma. For every pair $(k, j) \in \mathbb{N}^{2}$, we set the disjoint union $M_{k, j}:=K_{k+j-1} \cup G_{k+j}$, which is an RKL-set by (iii). Moreover, we can fix sequences $\left\{q_{j}: j \in \mathbb{N}\right\},\left\{Q_{k}: k \in \mathbb{N}\right\}$ which are dense in $\mathbb{C}$ and $L(\Omega)$, respectively.

Let us pick countably many pairwise disjoint infinite sets $J(k)(k \in \mathbb{N})$ such that $J(k) \subset\{j \in \mathbb{N}: j>k\}$ for all $k \in \mathbb{N}$ and, in addition, the sets $J(k)-k:=\{j-k: j \in J(k)\}(k \in \mathbb{N})$ are also mutually disjoint. This can be made in many ways; for instance, we may choose $J(k)=\left\{3^{\nu}-k^{2}: \nu>k\right\}$. We set $\mathcal{N}:=\bigcup_{k \in \mathbb{N}}(J(k)-k)$. In turn, we divide each $J(k)$ into infinitely many strictly increasing sequences $I(k, n)=\{p(k, n, l): l \in \mathbb{N}\}(n \in \mathbb{N})$. Then we have the disjoint union $\mathcal{N}:=\bigcup_{k, n \in \mathbb{N}}(I(k, n)-k)$. For every pair $(k, n) \in \mathbb{N}^{2}$, the mapping $\varphi(k, n, \cdot): j \mapsto l$-where $l$ is the unique positive integer with $k+j=p(k, n, l)$ - is a strictly increasing bijection from $I(k, n)-k$ onto $\mathbb{N}$. Therefore, trivially, the set $\left\{q_{\varphi(k, n, j)}: j \in I(k, n)-k\right\}$ is dense in $\mathbb{C}$.

Given $j \in \mathcal{N}$, there exist a unique $k=k(j) \in \mathbb{N}$ and also a unique $n=n(j) \in \mathbb{N}$ such that $j \in I(k, n)-k \subset J(k)-k$. From (a), there is $\delta_{j}>0$ such that

$$
\left\|T_{n} h\right\|_{F_{k+j}}<\frac{1}{2^{j}} \text { for all } h \in L(\Omega) \text { with }\|h\|_{G_{k+j}}<\delta_{j} .
$$

With no loss of generality, we may assume that the sequence $\left(\delta_{j}\right)$ is strictly decreasing and tends to zero. Then we can define $\tau_{j}:=\delta_{j}-\delta_{j+1}>0(j \geq 1)$. On the other hand, thanks to $(\mathrm{b})$, there exists a sequence $\left(\Phi_{n}\right) \subset L(\Omega)$ satisfying

$$
T_{n} \Phi_{n}=1 \text { for all } n \in \mathbb{N} \text {. }
$$

Fix now a number $k \in \mathbb{N}$. Inspired by the approach of [11] (see also [8]), we define $g_{k, 0}:=Q_{k}$ and $f_{k, 1}: M_{k, 1} \rightarrow \mathbb{C}$ as

$$
f_{k, 1}(x)= \begin{cases}g_{k, 0}(x) & \text { for every } x \in K_{k} \\ q_{l} \Phi_{n}(x) & \text { for every } x \in G_{k+1} \text { if } k+1=p(k, n, l) \in J(k) \\ 0 & \text { for every } x \in G_{k+1} \text { if } 1 \notin J(k)-k .\end{cases}
$$


Notice that $f_{k, 1}$ is well defined because if $1 \in J(k)-k$ then there is a unique $n \in \mathbb{N}$ with $1 \in I(k, n)-k$; so $k+1=p(k, n, l)$ for a unique $l \in \mathbb{N}$, namely, $l=\varphi(k, n, 1)$. Observe also that $f_{k, 1}$ is $L$-analytic in some neighborhood of $M_{k, 1}$. By Lemma 3.1, there is a function $g_{k, 1} \in L(\Omega)$ such that

$$
\left\|g_{k, 1}-f_{k, 1}\right\|_{M_{k, 1}}<\frac{\tau_{1}}{2^{k}}
$$

From this step, we proceed by induction. Define for $j \geq 2$ the function $f_{k, j}: M_{k, j} \rightarrow \mathbb{C}$ by

$$
f_{k, j}(x)= \begin{cases}g_{k, j-1}(x) & \text { for every } x \in K_{k+j-1} \\ q_{l} \Phi_{n}(x) & \text { for every } x \in G_{k+j} \text { if } k+j=p(k, n, l) \in J(k) \\ 0 & \text { for every } x \in G_{k+j} \text { if } j \notin J(k)-k .\end{cases}
$$

Again, $f_{k, j}$ is $L$-analytic in some neighborhood of the RKL-set $M_{k, j}$. Hence Lemma 3.1 guarantees the existence of a function $g_{k, j} \in L(\Omega)$ such that

$$
\left\|g_{k, j}-f_{k, j}\right\|_{M_{k, j}}<\frac{\tau_{j}}{2^{k}}
$$

Let us consider the series

$$
g_{k, 0}+\sum_{j=1}^{\infty}\left(g_{k, j}-g_{k, j-1}\right)
$$

For a prescribed compact subset $K \subset \Omega$, there is $m \in \mathbb{N}$ such that $K \subset$ $K_{k+m-1}$. Then for $j \geq m$ one has that

$$
\left\|g_{k, j}-g_{k, j-1}\right\|_{K} \leq\left\|g_{k, j}-f_{k, j}\right\|_{K_{k+j-1}} \leq\left\|g_{k, j}-f_{k, j}\right\|_{M_{k, j}}<\tau_{j},
$$

due to (3). But $\sum_{j \geq 1} \tau_{j}=\delta_{1}<\infty$, therefore (4) converges uniformly on compacta in $\Omega$. Thus, its sum defines a function $g_{k}$ belonging to $L(\Omega)$. Notice that, from the shape of (4), we have that

$$
g_{k}=\lim _{\nu \rightarrow \infty} g_{k, \nu}=g_{k, j}+\sum_{\nu=j+1}^{\infty}\left(g_{k, \nu}-g_{k, \nu-1}\right) \quad \text { for all } j \in \mathbb{N} .
$$

Finally, we define the desired linear submanifold $\mathcal{D}$ in $L(\Omega)$ as the linear span

$$
\mathcal{D}=\operatorname{span}\left\{g_{k}: k \in \mathbb{N}\right\}
$$


Let us fix a function $h \in L(\Omega)$, a compact set $K \subset \Omega$ and a number $\varepsilon>0$. Then by the exhaustion property (i) we get an $m \in \mathbb{N}$ with $K \subset K_{m}$; so $K_{k+j-1} \supset K$ for all $k \geq m$ and all $j \in \mathbb{N}$. Therefore, due to (3), (4) and the definition of $f_{k, j}$, we obtain for $k \geq m$ that

$$
\begin{aligned}
\left\|g_{k}-Q_{k}\right\|_{K} & =\left\|g_{k}-g_{k, 0}\right\|_{K} \leq \sum_{j=1}^{\infty}\left\|g_{k, j}-g_{k, j-1}\right\|_{K_{k+j-1}} \\
& \leq \sum_{j=1}^{\infty}\left\|g_{k, j}-f_{k, j}\right\|_{M_{k, j}}<\sum_{j=1}^{\infty} \frac{\tau_{j}}{2^{k}}=\frac{\delta_{1}}{2^{k}} .
\end{aligned}
$$

Since $\left\{Q_{k}: k \in \mathbb{N}\right\}$ is dense in $L(\Omega)$ there exists $k \geq m$ with $2^{k}>2 \delta_{1} / \varepsilon$ and $\left\|Q_{k}-h\right\|_{K}<\varepsilon / 2$. Hence, by the triangle inequality, $\left\|g_{k}-h\right\|_{K}<\varepsilon$. Thus, the set $\left\{g_{k}: k \in \mathbb{N}\right\}$ is dense in $L(\Omega)$. But $\mathcal{D} \supset\left\{g_{k}: k \in \mathbb{N}\right\}$, so $\mathcal{D}$ is also dense.

It remains to show that if a number $n \in \mathbb{N}$, a function $g \in \mathcal{D} \backslash\{0\}$ and a curve $\gamma \in \Gamma(\Omega)$ are prescribed, then $C_{\gamma}\left(T_{n} g\right)=\mathbb{C}^{*}$. For this, fix $n, g, \gamma$ as before. We can write $g=c_{1} g_{1}+\cdots+c_{k} g_{k}$ for certain $k \in \mathbb{N}$ and $c_{1}, \ldots, c_{k} \in \mathbb{C}$ with $c_{k} \neq 0$. Moreover, by the property (iv) we can select a number $j_{0} \in \mathbb{N}$ and points $x_{j} \in \gamma \cap F_{k+j}\left(j \geq j_{0}\right)$. Observe that the construction of the set $I(k, n)-k$ guarantees that for any $j \in I(k, n)-k$ the corresponding $k(j), n(j)$ for which (1) is satified are exactly our prescribed indexes $k, n$. Consequently, from (2), (5) and the linearity of $T_{n}$, we obtain for every $j \in I(k, n)-k$ with $j \geq j_{0}$ that

$$
\begin{aligned}
& \left|\left(T_{n} g\right)\left(x_{j}\right)-c_{k} q_{\varphi(k, n, j)}\right| \\
= & \left|T_{n}\left(c_{k} g_{k}+\sum_{i=1}^{k-1} c_{i} g_{i}\right)\left(x_{j}\right)-c_{k} q_{\varphi(k, n, j)} T_{n} \Phi_{n}\left(x_{j}\right)\right| \\
= & \mid c_{k} T_{n}\left(g_{k, j}-q_{\varphi(k, n, j)} \Phi_{n}\right)\left(x_{j}\right)+c_{k} T_{n}\left(\sum_{\nu=j+1}^{\infty}\left(g_{k, \nu}-g_{k, \nu-1}\right)\right)\left(x_{j}\right) \\
& +\sum_{i=1}^{k-1} c_{i} T_{n}\left(g_{i}\right)\left(x_{j}\right) \mid \\
\leq & \left\|c_{k} T_{n}\left(g_{k, j}-q_{\varphi(k, n, j)} \Phi_{n}\right)+c_{k} T_{n}\left(\sum_{\nu=j+1}^{\infty}\left(g_{k, \nu}-g_{k, \nu-1}\right)\right)+\sum_{i=1}^{k-1} c_{i} T_{n} g_{i}\right\|_{F_{k+j}}
\end{aligned}
$$




$$
\begin{gathered}
\leq\left|c_{k}\right|\left\|T_{n}\left(g_{k, j}-q_{\varphi(k, n, j)} \Phi_{n}\right)\right\|_{F_{k+j}}+\left|c_{k}\right|\left\|T_{n}\left(\sum_{\nu=j+1}^{\infty}\left(g_{k, \nu}-g_{k, \nu-1}\right)\right)\right\|_{F_{k+j}} \\
+\sum_{i=1}^{k-1}\left|c_{i}\right|\left\|T_{n} g_{i}\right\|_{F_{k+j}}
\end{gathered}
$$

First, we get from (3) that $\left\|g_{k, j}-q_{\varphi(k, n, j)} \Phi_{n}\right\|_{G_{k+j}}<\tau_{j}\left(<\delta_{j}\right)$. Hence by $(1)$ it is derived that

$$
\left\|T_{n}\left(g_{k, j}-q_{\varphi(k, n, j)} \Phi_{n}\right)\right\|_{F_{k+j}}<\frac{1}{2^{j}} .
$$

Second, since $G_{k+j} \subset K_{k+j} \subset K_{k+\nu-1}$ for all $\nu \geq j+1$ and $g_{k, \nu-1}=f_{k, \nu}$ on $K_{k+\nu-1}$, one deduces via (3) that $\left\|g_{k, \nu}-g_{k, \nu-1}\right\|_{G_{k+j}}<\tau_{\nu}$. Hence, by the triangle inequality,

$$
\left\|\sum_{\nu=j+1}^{\infty}\left(g_{k, \nu}-g_{k, \nu-1}\right)\right\|_{F_{k+j}}<\sum_{\nu=j+1}^{\infty} \tau_{\nu}=\delta_{j+1}<\delta_{j} .
$$

Thus, again from (1), we obtain

$$
\left\|T_{n}\left(\sum_{\nu=j+1}^{\infty}\left(g_{k, \nu}-g_{k, \nu-1}\right)\right)\right\|_{F_{k+j}}<\frac{1}{2^{j}} .
$$

Next, each quantity $\left\|T_{n} g_{i}\right\|_{F_{k+j}}(i=1, \ldots, k-1)$ should be estimated. This is a bit more involved. According to (5), we can expand $g_{i}$ as

$$
g_{i}=g_{i, k-i+j}+\sum_{\nu=k-i+j+1}^{\infty}\left(g_{i, \nu}-g_{i, \nu-1}\right)
$$

Analogously to the second estimation, observe that $G_{k+j} \subset K_{k+j} \subset K_{i+\nu-1}$ for all $\nu \geq k+j-i+1$, and $g_{i, \nu-1}=f_{i, \nu}$ on $K_{i+\nu-1}$. Therefore, due to (3), $\left\|g_{i, \nu}-g_{i, \nu-1}\right\|_{G_{k+j}}<\tau_{\nu}$. Furthermore,

$\left\|g_{i, k-i+j}\right\|_{G_{k+j}}=\left\|g_{i, k-i+j}-f_{i, k-i+j}\right\|_{G_{i+k-i+j}}<\left\|g_{i, k-i+j}-f_{i, k-i+j}\right\|_{M_{i, k-i+j}}<\tau_{k-i+j}$.

This holds because of (3) and of the fact that $f_{i, k-i+j}=0$ on $G_{k+j}$, which in turn is true because $k+j \in J(k)$, so $i+(k-i+j)=k+j \notin J(i)$ (recall 
that the sets $J(\nu)$ 's are pairwise disjoint). The triangle inequality drives us to

$$
\left\|g_{i}\right\|_{G_{k+j}}<\tau_{k-i+j}+\sum_{\nu=k-i+j+1}^{\infty} \tau_{\nu}=\tau_{k-i+j}+\delta_{k-i+j+1}=\delta_{k-i+j}<\delta_{j} .
$$

Once more, (3) tells us that

$$
\left\|T_{n} g_{i}\right\|_{F_{k+j}}<\frac{1}{2^{j}} \quad(i=1, \ldots, k-1) .
$$

Putting (6), (7) and (8) together and setting $M:=2 \sum_{i=1}^{k}\left|c_{i}\right|<\infty$, we finally get

$$
\left|\left(T_{n} g\right)\left(x_{j}\right)-c_{k} q_{\varphi(k, n, j)}\right|<\frac{M}{2^{j}} \rightarrow 0(j \rightarrow \infty) .
$$

As a final step, let us fix $c \in \mathbb{C}$ and choose an increasing sequence $\{j(1)<$ $j(2)<\cdots\} \subset I(k, n)-k$ such that $q_{\varphi(k, n, j(s))} \rightarrow c / c_{k}$ as $s \rightarrow \infty$. Observe that $x_{j(s)}(\in \gamma)$ tends to $\partial \Omega$. By using $(9)$ we get $\lim _{s \rightarrow \infty}\left(T_{n} g\right)\left(x_{j(s)}\right)=$ c. Consequently, $c \in C_{\gamma}\left(T_{n} g\right)$ for all $c \in \mathbb{C}$. Thus, $C_{\gamma}\left(T_{n} g\right)=\mathbb{C}^{\star}$, as desired.

Note that thanks to the Examples 2.2 our Theorem 4.1 contains and strengthens both results [3, Theorem 5] and [1, Theorem 2.1] mentioned in the Introduction. Indeed, for the first case take as $\left(T_{n}\right)$ a sequence whose members are all partial derivations $\partial^{\alpha}$, and for the second case take $N=2$, $\Omega=$ a Jordan domain, $L=\bar{\partial}$, and $T_{n}=$ the identity $(n \in \mathbb{N})$.

As a final remark, we point out that, at least for $L=\Delta$ in $\mathbb{R}^{N}$ and $L=\bar{\partial}$ in $\mathbb{R}^{2}$, our theorem is close to being sharp. Indeed, it is shown in $[3$, Proposition 4] that if $\Omega$ is a domain in $\mathbb{R}^{N}$ such that its $\left(\mathbb{R}^{N}\right)^{*}$-boundary has an isolated point $b$, then for each function $f$ harmonic in $\Omega$ or (if $N=2$ ) for each function $f$ holomorphic in $\Omega$, there exists an admissible path $\gamma \subset \Omega$ ending at $b$ such that $\mathcal{C}_{\gamma}(f)$ is a single point in $\mathbb{C}^{*}$. 


\section{References}

[1] L. Bernal-González, M.C. Calderón-Moreno and J.A. Prado-Bassas, Maximal cluster sets along arbitrary curves, J. Approx. Theory 129 (2004), 207-216.

[2] R.P. Boas, Entire functions, Academic Press, New York, 1954.

[3] A. Boivin, P.M. Gauthier and P.V. Paramonov, Approximation on closed sets by analytic or meromorphic solutions of elliptic equations and applications, Canadian J. Math. 54 (2002), 945-969.

[4] A. Boivin, and P.V. Paramonov, Approximation by meromorphic and entire solutions of elliptic equations in Banach spaces of distributions, Math. Sb. (4) 189 (1998), 481-502.

[5] E.F. Collingwood and A.J. Lohwater, The theory of cluster sets, Cambridge University Press, Londres, 1966.

[6] A. Dufresnoy, P.M. Gauthier and W.H. Ow, Uniform approximation on closed sets by solutions of elliptic partial differential equations, Complex Variables Theory Appl. 6 (1986), 235-247.

[7] D. Gaier, Lectures on Complex Approximation, London Math. Society Lecture Notes 221, Cambridge Univ. Press, 1995.

[8] P.M. Gauthier, Tangential approximation and universality, J. Contemp. Math. Anal. 36 (2001), 2-13.

[9] K. Noshiro, Cluster sets, Springer-Verlag, Berlin, 1960.

[10] G.E. Shilov, Generalized functions and partial differential equations, Gordon and Breach, New York, 1968.

[11] A. Sinclair, A general solution for a class of approximation problems, Pacific J. Math. 8 (1958), 857-866.

[12] F. Trèves, Linear partial differential equations with constant coefficients, Gordon and Breach, New York, 1966. 
L. Bernal-González, M.C. Calderón-Moreno Departamento de Análisis Matemático Facultad de Matemáticas, Apdo. 1160

Avda. Reina Mercedes, 41080 Sevilla, Spain

E-mails: lbernal@us.es, mccm@us.es

Antonio Bonilla

Departamento de Análisis Matemático

Universidad de La Laguna

C/Astrofísico Fco. Sánchez, s/n

38271 La Laguna, Tenerife, Spain

E-mail: abonilla@ull.es

J.A. Prado-Bassas

Departamento de Matemáticas

Universidad Autónoma de Madrid

Ctra. Colmenar Viejo Km. 15

28049, Madrid, Spain

E-mail: joseantonio.prado@uam.es 